\title{
Assessing the Power of Intensity Interaction between the Solid and Fluid Phases in the Unconsolidated Water-Saturated Sandy Marine Sediments at Shear Wave Propagation
}

\author{
V. A. Lisyutin ${ }^{\bowtie}$, O. R. Lastovenko \\ Sevastopol State University, Sevastopol, Russian Federation \\ 凶vlisiutin@mail.ru
}

Purpose. Propagation of a shear wave in sandy marine sediments is considered. The acoustic properties of a shear wave are the phase velocity and the attenuation coefficient. It is known that in dry sandy sediments, the attenuation coefficient is directly proportional to frequency. In the saturated mediums, there are the deviations from this law that implies existence of two physical mechanisms of losses - the intergranular friction and viscous loss. The study is aimed at developing a two-phase theoretical model of the shear wave propagation in the unconsolidated marine sediments, and at identifying the dissipative effects caused by the fluid relative movement in the pore space.

Methods and Results. The intergranular friction is modeled using a springpot, which represents an element combing conservative properties of a spring and dissipative ones of a dashpot. The equation of motion is applied, where a part of fluid is assumed to be associated with the media solid phase and another part is considered to be mobile. For a harmonic displacement, the equations of state and the equation of motion yield a new two-phase dispersion relation (the theory of Grain Shearing + Effective Density, or GS + EDs, for short). The results of the GS + EDs theory are compared with the data of the velocity and attenuation measurements taken from the open sources. It is shown that during propagation of the compressional and shear waves, the mechanisms of interaction between the granules, and between the granules and fluid are not similar. Character of the changes in the grain-to-grain friction parameters when the pore space is saturated with fluid, is analyzed.

Conclusion. Manifestation of the dissipative effects resulting from the pore saturation with fluid depends on the density of the granules packing. In case of a dense packing, there are no conditions for the fluid relative movement, and the sandy sediments exhibit the property of constant $Q$-factor. If the packing is loose, the viscous losses make a significant contribution, and the attenuation frequency dependence is nonlinear. The effective pore sizes for the compression and shear waves do not coincide.

Keywords: unconsolidated marine sediments, intergranular friction, viscous losses, dispersion, shear wave, attenuation coefficient, dispersion relation

Acknowledgements: the investigation was carried out within the framework of the Sevastopol State University internal grant "Development of theoretical models for physical methods of research of the Black Sea shelf", project No. 41/06-31.

For citation: Lisyutin, V.A. and Lastovenko, O.R., 2021. Assessing the Power of Intensity Interaction between the Solid and Fluid Phases in the Unconsolidated Water-Saturated Sandy Marine Sediments at Shear Wave Propagation. Physical Oceanography, [e-journal] 28(1), pp. 90-103. doi:10.22449/1573-160X-2021-1-90-103

DOI: 10.22449/1573-160X-2021-1-90-103

(C) V. A. Lisyutin, O. R. Lastovenko, 2021

(C) Physical Oceanography, 2021

Introduction. In rocks, soils and marine sediments, not only longitudinal, but also transverse (shear) waves can propagate [1, 2]. In consolidated media such as shell rocks and sandstones, the connection between particles of the solid phase during shear deformation appears due to the elastic skeletal frame existence. 
In unconsolidated marine sediments such as sand, silt and clay it arises due to intergranular (internal, grain-to-grain shearing) friction.

The results of measurements in dry rock formations and sands show a linear frequency dependence of the attenuation coefficient $(\mathrm{dB} / \mathrm{m})$ of a shear wave: $\alpha_{\mathrm{s}}=\alpha_{\mathrm{s} 0} f^{\delta}$, where $\delta \approx 1$ is the attenuation exponent [3-5]. On the other hand, R.D. Stoll [6], B. Brunson [7], M. Kimura [8, 9] and N. Chotiros [10, 11] pay attention to deviations of the exponent $\delta$ from unity in water-saturated media.

Linear dependence of the attenuation coefficient is explained by internal friction between particles of the solid phase of marine sediments, deviations from linearity and significant dispersion of the shear wave velocity in the vicinity of the transition frequency - by viscous losses during the relative motion of the pore fluid.

The exponent $\delta$ in the power law frequency dependence of the attenuation coefficient reflects the nature of the interaction between the solid and fluid phases of the medium. If $\delta=1$, the phases do not interact - this is a two-component medium. If $1<\delta<2$, which is below the transition frequency, the fluid phase slips and in the pores - the Poiseuille flow, viscous forces dominate. If $0.5<\delta<1$, which is above the transition frequency, the fluid slips, the relative velocity profile is flat - inertial forces dominate.

In $[12,13]$, the Grain Shearing + Effective Compressibility (GSEC) theory is presented, considering the propagation of a longitudinal wave in marine sediments. The GSEC model takes into account grain shearing (GS) friction and viscous losses. To attach viscous losses in the formula for the phase velocity of sound, the equilibrium compressibility of the medium is replaced with an effective (twophase) modulus that depends on frequency, resulting in an effective compressibility (EC) type dispersion that affects only the longitudinal wave. Mass property of the medium - the density is assumed constant.

The work [14] represents the GS + EDensity model, in which, in the formula for the phase velocity of sound, the compressibility of the medium is assumed to be constant, but the equilibrium density is replaced by an effective complex one, giving a dispersion of the inertial-viscous type that affects both types of waves longitudinal and transverse.

Both models show the same calculation result. In a real medium, these types of dispersion can appear in varying power.

Variation in the mass properties of the medium can be estimated by analyzing the results of measurements of the frequency dependences of the shear wave attenuation. The power of interaction of solid and fluid phases in water-saturated unconsolidated sediments during shear is a controversial issue. There are two opposite points of view. R.D. Stoll [6], B. Brunson [7], M. Kimura [8, 9] and $\mathrm{N}$. Chotiros $[10,11]$ argue that shear wave attenuation in marine sediments changes with frequency $(\omega=2 \pi f)$ according to a nonlinear law, and E. Hamilton [5] and M. Buckingham $[15,16]$ believe that the attenuation coefficient $\alpha_{\mathrm{s}} \sim \omega^{1}$.

Answering this question, it is possible to identify the sources of longitudinal wave dispersion too - a change in the compressibility of the medium $[12,13]$, or a change in its effective density [14], or both (in this case, their proportion). The present article is a continuation of the research [12-14]. 
Dispersion relation for a shear wave. The propagation of a shear wave in unconsolidated marine sediments is possible only due to the forces of intergranular friction. On the other hand, friction will cause energy losses also.

Below the shear wave of $u_{y g}=\exp \left(i \omega t-i k_{s} x\right)$ type, propagating along the $O X$ axis in a porous fluid-saturated medium is considered. The transverse displacement of particles of the solid phase is denoted by $u_{y g}$ and the wavenumber by $k_{\mathrm{s}}$.

The interaction between the granules will be modeled by a connection through a springpot - an element that combines the conservative properties of a spring and the dissipative properties of a dashpot [17]. The springpot state equation assumes a viscoelastic-plastic deformation and has the form as follows

$$
\sigma(t)=\mu \tau^{m} \frac{\partial^{m} \varepsilon}{\partial t^{m}},
$$

where $\sigma$ is the shear tension; $\mu$ and $\tau$ are the constants, reflecting the balance between energy accumulation and dissipation; $m$ is the order of the fractional derivative determining the value of the attenuation exponent $\delta, 0<m<1$; $\varepsilon=\frac{\partial u_{y g}}{\partial x}-$ is the shear strain $\left(\frac{\partial u_{y g}}{\partial x}\right.$ is the divergence component). In case $m=1$, the springpot transforms into dashpot and the oscillatory process will be damped and non-wave; in case $m \rightarrow 0$, then the springpot is a spring and the oscillations will be undamped.

Pore space of a real medium has a complex structure consisting of variable cross-section sections. Small granules can clog pore passages, creating isolated cavities. Fluid trapped in such cavities will oscillate with the granules.

Let $P$ - is static porosity of the medium, corresponding to the volume of the fluid, containing all the pores; $\rho_{f}, \rho_{g}$ are densities of the fluid and solid phase, respectively. The fraction of fluid moving relative to granules will be characterized by the effective (percolation [12]) porosity $\varphi$.

Thus, the mass fraction of fluid equal to $(P-\varphi) \rho_{f}$ oscillates together with the fraction of the solid phase equal to $(1-P) \rho_{g}$, and only the fraction of the fluid $\varphi \rho_{f}$ is entrained by the granules as a result of inertial-viscous interaction and oscillates separately with a smaller amplitude. The equation of motion can be written in the following form

$$
\frac{\partial^{2}}{\partial t^{2}}\left((1-P) \rho_{g} u_{y g}+(P-\varphi) \rho_{f} u_{y g}+\varphi \rho_{f} U_{y f}\right)=\frac{\partial \sigma}{\partial x},
$$

where $U_{y f}$ is the displacement of fluid.

Applying $\partial / \partial x$ to (1), comparing with (2), we obtain

$$
\rho_{m} \dot{u}_{y g}-\varphi \rho_{f}\left(\dot{u}_{y g}-\dot{U}_{y f}\right)=\mu \tau^{m} \frac{\partial^{m}}{\partial t^{m}} \frac{\partial^{2} u_{y g}}{\partial x^{2}},
$$

where $\rho_{m}=P \rho_{f}+(1-P) \rho_{g}$ is the equilibrium density of the medium. 
By defining the complex phase velocity as $\widetilde{c}_{\mathrm{s}}=\omega / k_{\mathrm{s}}$, substituting the harmonic dependence into (3), we obtain the equation for the phase velocity

$$
\widetilde{c}_{\mathrm{s}}=\sqrt{\frac{\gamma_{s}\left(i \omega t_{0}\right)^{m}}{\rho_{m}-\varphi \rho_{f}\left(1-U_{\mathrm{av}}\right)}},
$$

Where $\gamma_{\mathrm{s}}=\left(\tau / t_{0}\right)^{m} \mu$ is the shear rigidity modulus, $\mathrm{Pa} ; t_{0}=1 \mathrm{~s}$ is the formal constant, restoring correct physical dimension; $U_{\mathrm{av}}$ is average over the cross section of the pore amplitude of fluid oscillations.

Considering a medium with pores in the form of cylindrical tubes of radius $a$, the axis of which is oriented along the vector $\mathbf{u}$ of the solid phase displacement, solving the Navier - Stokes equation [18], the value $\left(1-U_{\mathrm{av}}\right)$ can be calculated

$$
1-U_{\mathrm{av}}=1-\frac{2 J_{1}\left(i^{3 / 2} w\right)}{i^{3 / 2} w J_{0}\left(i^{3 / 2} w\right)}=F_{C}(w),
$$

here $J_{0}, J_{1}$ are the Bessel functions;

$$
w=\sqrt{\frac{a^{2} \rho_{f}}{\eta} 2 \pi f},
$$

where $\eta$ is dynamic viscosity of the fluid, Pa.s. Notice that the expressions for frequency correction function $F_{C}$ and its argument in [12, p. 82; 13, p. 404; 14, p. 43], coincide with formulas (5) and (6). Graph of the correction function versus frequency $f$ normalized to the transition frequency $f_{r}=\sqrt{\eta / a^{2} \rho_{f}}$, is shown in Fig. 1.

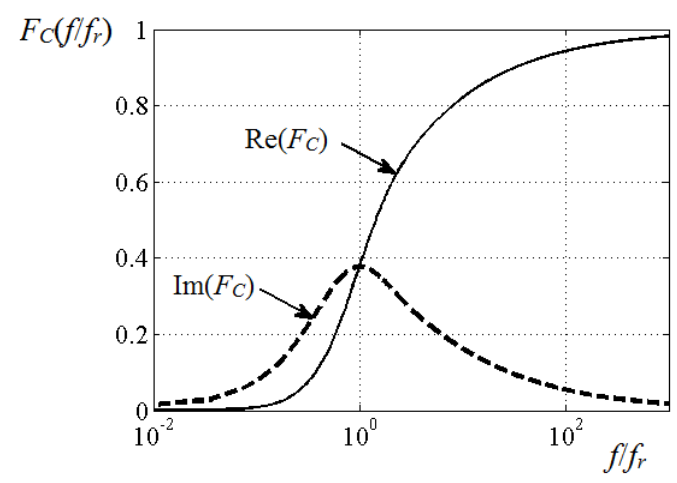

F i g. 1. Frequency correction function depending on the normalized frequency
Substituting (5) in (4), the dispersion relation for the shear wave in the following form is obtained

$$
\widetilde{c}_{\mathrm{s}}=\sqrt{\frac{\gamma_{\mathrm{s}}\left(i \omega t_{0}\right)^{m}}{\rho_{\text {seff }}}},
$$

where

$$
\rho_{\text {seff }}=\rho_{m}-T_{\mathrm{s}}^{-1} \varphi \rho_{f} F_{C}(w)
$$

Is the shear effective density; $T_{\mathrm{s}}$ is the pore tortuosity factor $[6,13]$.

The real phase speed $c_{\mathrm{s}}$ and attenuation coefficient $\alpha_{\mathrm{s}}$ can be obtained from the complex velocity: $c_{\mathrm{s}}=\left(\operatorname{Re}\left({\widetilde{c_{s}}}^{-1}\right)\right)^{-1} ; \alpha_{\mathrm{s}}=-\omega \operatorname{Im}\left(\widetilde{c}_{\mathrm{s}}^{-1}\right), \mathrm{Np} / \mathrm{m}$.

The real part of the second term of effective density (8) characterizes the inertial interaction of phases and controls the phase velocity dispersion, PHYSICAL OCEANOGRAPHY VOL. 28 ISS. 1 (2021) 
the imaginary part characterizes viscous losses and controls the attenuation. Effective density has a simple physical interpretation. At low frequencies, $\operatorname{Re}\left(F_{C}\right) \rightarrow 0, \operatorname{Im}\left(F_{C}\right) \rightarrow 0, \rho_{\text {seff }} \approx \rho_{m}$, almost all of the fluid is entrained by the oscillating granules, and the viscous forces are negligible. In the vicinity of the transition frequency, a part of the fluid is entrained and a part slips, and the viscous forces are maximum. At high frequencies, the granules and the fluid trapped in the pores oscillate together, the transversely movable part of the fluid slips. Viscous forces are concentrated in a thin boundary layer around the contour of through pores.

In case $\varphi=0$, then the dispersion relation (7) coincides with the corresponding relation of the GS theory developed by $M$. Buckingham [16, p. 2812]. The model of intergranular interaction in the form of a springpot is equivalent to the phenomenon of strain hardening - an increase in the resistance of the dashpot with increasing deformation $[15,16]$.

The presented model will be referred to below as GSEDs, noting its application to shear wave propagation.

Comparison of theoretical results with experimental data. Energy losses during the propagation of elastic waves in media are usually characterized by the attenuation coefficient $\alpha(\mathrm{dB} / \mathrm{m})=8.69 \cdot \alpha(\mathrm{Np} / \mathrm{m})$. When calculating acoustic fields, the loss tangent $\beta_{\mathrm{s}}$ is often used. It is included in the formula for the wave number $k_{\mathrm{s}}=\frac{\omega}{c_{\mathrm{s}}}\left(1-i \beta_{\mathrm{s}}\right), \beta_{\mathrm{s}}=\frac{\alpha_{\mathrm{s}} c_{\mathrm{s}}}{\omega}$. In geophysics, the quality factor $Q_{\mathrm{s}}=1 /\left(2 \beta_{\mathrm{s}}\right)$ is often used.

To calculate the loss tangent or quality (Q) factor, it is necessary to have measurements of both the speed and the attenuation coefficient at the same frequencies. If only the attenuation was measured at different frequencies, then its deviations from the linear dependence are most clearly manifested in the difference between the specific attenuation coefficient $\alpha_{\mathrm{s} 0}(\mathrm{~dB} /(\mathrm{m} \cdot \mathrm{kHz}))$ from a constant value or the attenuation exponent $\delta$ from a unit.

The input GSEDs model parameters that characterize grain-to-grain shearing are the shear rigidity modulus $\gamma_{s}, \mathrm{~Pa}$, and the strain hardening exponent $m$. These two parameters are determined by the experimental data inversion. If the motion of the fluid is neglected, then the attenuation exponent $\delta$ and strain hardening exponent are related by the ratio $\delta=1-m / 2$.

The input parameters characterizing the pore space and fluid movement are as follows: $a$ is a pore size; $\varphi$ is an effective porosity. These parameters can be determined by comparing the model dependence $\alpha_{s 0}(f)$ with the measured one.

An a priori estimate of the pore size during the longitudinal wave propagation can be obtained using frequency dependence measurements of the permeability of the medium composed of identical glass beads [13]:

$$
a=d_{e} / 7,35=0,136 d_{e},
$$

where $d_{e}$ is the diameter of beads that make up an equivalent medium, the permeability of which is equal to the measured permeability of the real medium. The size of the beads corresponding to the measured permeability can be 
calculated by inverting the Kozeny-Carman's relation $\kappa_{0}=\frac{1}{180} \frac{P^{3}}{(1-P)^{2}} d^{2}$, where $\kappa_{0}$ is the steady-state permeability of the medium, $\mathrm{m}^{2}$. Taking into account that the porosity of the medium from beads $P_{0}$ with random packing is $\sim 0.37$, knowing the real medium permeability, it is possible to calculate the equivalent diameter of the beads:

$$
d_{e}=39 \sqrt{\kappa_{0}} .
$$

Note that the in situ measurements used below do not allow inverting the dependence of friction microparameters on depth, which arises due to the presence of vertical gradients of the physical properties of sediments (porosity, permeability), as well as geostatic pressure. Within the framework of the one-phase theory of intergranular friction, this issue is studied by M. Buckingham in [19].

Below the results of the experiments carried out by B. Brunson [7, 15, 20], D. Bell [10, 15, 21], M. Kimura [8, 9] and SAX99 (Sediment Acoustics Experiment 1999) [22, 23] (Fig. 2, table) are considered.

B. Brunson [7] measured the attenuation coefficient $\alpha_{s 0}(f)$ in water-saturated angular sand and glass beads in the frequency range from 1 to $20 \mathrm{kHz}$, and the shear wave velocity $c_{\mathrm{s}}(f)$ - at one frequency. The granular medium was contained in a vertical acrylic cylinder about $0.5 \mathrm{~m}$ long, and a pressure of $12 \mathrm{kPa}$ was applied from above, corresponding to a depth of $1.35 \mathrm{~m}$. B. Brunson and R. Johnson [20] carried out similar measurements with quartz sand in the frequency range 0.45 to $7 \mathrm{kHz}$ at a vertical pressure of $3.5 \mathrm{kPa}$ (depth $\sim 0.4 \mathrm{~m}$ ) (Fig. 2, $a, 2, b$; table). In Fig. 2, $a, 2, b$, experimental points (blue diamonds) also show the results of inversion of the shear wave velocity and attenuation, obtained in situ during measurements of the dispersion-dissipative properties of the surface wave at low frequencies of 20, 25 and $35 \mathrm{~Hz}$ [15]. These three points are located on an almost vertical axis. In the same figure, the measurement results obtained in the SAX99 marine experiment $\left(c_{s}=120 \mathrm{~m} / \mathrm{s}, \alpha_{s}=30 \mathrm{~dB}, f=1 \mathrm{kHz}\right)[22,23]$. SAX99 location is clean sand with granules of $0.29-0.56 \mathrm{~mm}$ in size.

In his experiments $\mathrm{D}$. Bell measured the wave velocity $c_{\mathrm{s}}\left(f_{0}\right)$ at one frequency and its attenuation $\alpha_{s}(f)$ in the frequency range $0.6-20 \mathrm{kHz}$ in sand and small glass beads, being water-saturated and dry (Fig. 2a, 2b; table). The measurements were carried out in small $(16 \times 20 \times 30 \mathrm{~cm})$ and large $(46 \times 56 \times 70 \mathrm{~cm}$, volume $150 \mathrm{~L})$ boxes. The sensors were recessed into the medium by $20 \mathrm{~cm}$, which corresponds to a pressure of $1.8 \mathrm{kPa}$ [21].

M. Kimura [8, 9] measured the shear wave speed and attenuation at various frequencies in sorted sand (Fig. 2, c - f; table). A desk-size measuring setup was used; sand samples were packed in polyethylene cylinders $43 \mathrm{~mm}$ in diameter and up to $200 \mathrm{~mm}$ in height. The external load caused a pressure of $17.3 \mathrm{kPa}$, which corresponds to a depth of $1.75 \mathrm{~m}$. The measurements were carried out in sieved sand with the same grain size at different temperatures and with different grain sizes - at the same temperature. 

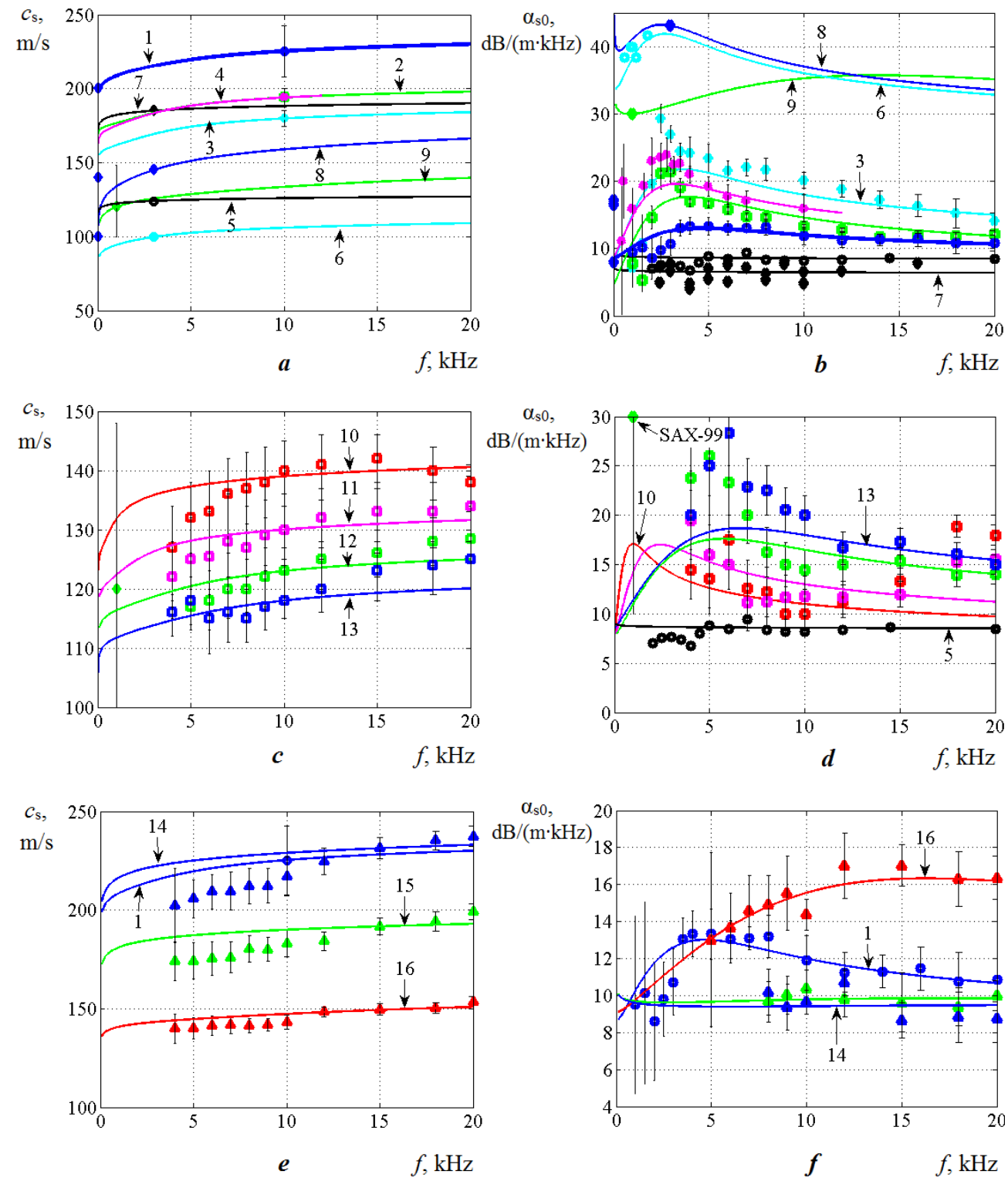

$\mathbf{F} \mathbf{i}$ g. 2. Frequency dependences of the phase velocity $c_{\mathrm{s}}(a, c, e)$ and the attenuation coefficient $a_{\mathrm{s} 0}$ $(b, d, f)$ of the shear wave: $a, b$ - in the measurements of B. Brunson and R. Johnson [7, 20] (green squares - in angular sieved sand; blue stars - in non-sieved sand based on the data from [7]; pink stars - in non-sieved sand based on the data from [20]; blue circles - in glass beads) and D. Bell [21] (black rhombuses - in dry sand; blue diamonds - in water-saturated sand; black circles - in dry glass beads; blue circles - in water-saturated beads); $c, d$ - in the measurements of $\mathrm{M}$. Kimura in the sorted sand with different granule sizes at temperature $20^{\circ} \mathrm{C}$ and $\mathrm{D}$. Bell in dry glass beads; $e, f-$ in the measurements of $\mathrm{M}$. Kimura in sorted sand at various temperature. The experimental points are indicated by symbols, the calculation graphs - by numerals (see the table) 
In the results of measurements carried out at different times using different installations, at high frequencies $(12 \mathrm{kHz})$, a change in attenuation from $9(\mathrm{~dB} /(\mathrm{m} \cdot \mathrm{kHz})$ for large granules to $17 \mathrm{~dB} /(\mathrm{m} \cdot \mathrm{kHz})$ for small granules is noted (Fig. 2). An exception is the results of measurements carried out by D. Bell and the SAX99 research group at the lowest external pressure. A significant difference is observed in the shear wave velocities. The best exemplary agreement between the experimental points, the theoretical dependence $\alpha_{s 0}(f)$ and the inverted parameters of the pore space show the results of B. Brunson's experiments in a medium of glass beads (blue circles, graph 1 in Fig. 2, $a, 2, b$; table).

The input physical parameters and the intergranular friction and pore space parameters reconstructed based on the data of different experiments

\begin{tabular}{|c|c|c|c|c|c|c|c|c|c|}
\hline \multirow{3}{*}{ Parameter } & \multicolumn{4}{|c|}{ B. Brunson and R. Johnson $[7,20]$} & \multicolumn{4}{|c|}{ D. Bell [21] } & \multirow[b]{3}{*}{ Sand in sea } \\
\hline & \multirow[b]{2}{*}{$\begin{array}{c}\text { Glass beads } \\
{[7]}\end{array}$} & \multicolumn{3}{|c|}{ Sand } & \multicolumn{2}{|r|}{ Beads } & \multicolumn{2}{|r|}{ Sand } & \\
\hline & & sieved / [7] & non-sieved [7] & $\begin{array}{c}\text { non-sieved } \\
{[20]}\end{array}$ & dry & $\begin{array}{l}\text { water- } \\
\text { saturated }\end{array}$ & dry & water-saturated & \\
\hline & 1 & 2 & 3 & 4 & 5 & 6 & 7 & 8 & 9 \\
\hline$d, \mathrm{~mm}$ & 0.385 & 0.385 & 0.372 & 0.35 & \multicolumn{2}{|r|}{0.177} & \multicolumn{2}{|r|}{0.354} & 0.379 \\
\hline$d_{e}, \mathrm{~mm}$ & 0.416 & 0.372 & 0.332 & $0.4^{*}$ & - & \begin{tabular}{|l|}
0.16 \\
\end{tabular} & - & 0.24 & $0.176[13]$ \\
\hline$P$ & 0.355 & 0.432 & 0.432 & 0.395 & $|-|$ & $0.365 \pm 0.008$ & - & $0.386 \pm 0.014$ & $0.372[13]$ \\
\hline$\eta$ & \multicolumn{9}{|c|}{ accepted equal to $1 \cdot 10^{-3} \mathrm{~Pa} \cdot \mathrm{s}$} \\
\hline$\rho_{w}, \mathrm{~kg} / \mathrm{m}^{3}$ & 1968 & 1937 & 1937 & 1998 & $1550 \pm 2$ & 1920 & 1600 & 1990 & 2069 \\
\hline$\kappa_{0} \cdot 10^{-11}, \mathrm{~m}^{2}$ & 11.43 & 9.08 & 7.25 & $10.5^{* *}$ & - & 1.7 & - & 3.79 & 2.02 \\
\hline$T$ & $1.65^{* * *}$ & $1^{* *}(1.92[7])$ & $1^{* * *}(2.52[7])$ & $1^{\cdots *}$ & 1.65 & 1.65 & - & $1.3^{\cdots *}$ & 1.35 \\
\hline$\left\langle\alpha_{s 0}\right\rangle, \mathrm{dB} / \mathrm{m} \cdot \mathrm{kHz}$ & $11.48 \pm 1.50$ & $14.4 \pm 4.3$ & $19.6 \pm 5.7$ & $19.5 \pm 3.7$ & 8.1 & $33 \pm 6$ & $6.0 \pm 1.4$ & 43 & $30 \pm 10$ \\
\hline$\alpha_{p 0}, \mathrm{~dB} / \mathrm{m} \cdot \mathrm{kHz}$ & - & - & - & - & 109 & 0.23 & 23 & 0.23 & $0.4[13]$ \\
\hline$\gamma_{s} \cdot 10^{6}, \mathrm{~Pa}$ & 60.5 & 51.0 & 39.0 & 45.2 & 18.4 & 9.43 & 41.5 & 13.6 & 14.8 \\
\hline$\gamma \cdot 10^{6}, \mathrm{~Pa}$ & $190[13]$ & - & - & - & 1.24 & 164.2 & 15.5 & 61.9 & 23.6 [13] \\
\hline$m$ & 0.039 & 0.018 & 0.0284 & 0.033 & 0.025 & 0.067 & 0.0284 & 0.11 & 0.08 \\
\hline$n$ & $0.06[13]$ & - & - & - & 0.25 & 0.092 & 0.12 & 0.13 & $0.192[13]$ \\
\hline$\varphi_{s} ; \varphi_{p}$ & $0.355 ; 0.3[13]$ & 0.432 & 0.432 & 0.395 & $0.365 ; 0$ & $\begin{array}{c}0.365 ; 0.23 \\
{[13]}\end{array}$ & 0.386 & $0.386 ; 0.23[13]$ & $0.372 ; 0.23$ \\
\hline$d_{e} / a_{\mathrm{s}}$ & 29 & 23 & 20 & 23 & & 9 & & 14 & 23 \\
\hline$d_{e} / a_{p}$ & $7.35[13]$ & - & - & - & - & & - & & $7.35[13]$ \\
\hline
\end{tabular}

${ }^{*}$ The value is calculated by formula $d_{e}=23 \cdot a_{\mathrm{s}}, a_{\mathrm{s}}=17.4 \mu \mathrm{m}$ and Fig. $2, b$ (the maximum value is on graph $\left.\alpha_{\mathrm{s} 0}(f)\right)$, and by the formula for transition frequency $f_{r}$.

${ }^{* *}$ It was not measured instrumentally, but was calculated by the inverted formula (10) with substitution $d_{e}=d$.

${ }^{* * * *}$ The tortuosity coefficient was determined from the best fit of graph $\alpha_{\mathrm{s} 0}(f)$ to the experimental points. 
Continuation of the table

\begin{tabular}{|c|c|c|c|c|c|c|c|}
\hline \multirow{4}{*}{ Parameter } & \multicolumn{7}{|c|}{ M. Kimura } \\
\hline & \multicolumn{4}{|c|}{ At temperature $20^{\circ} \mathrm{C}[9]$} & \multicolumn{3}{|c|}{ At different temperature [8] } \\
\hline & & & & & $5^{\circ} \mathrm{C}$ & $20{ }^{\circ} \mathrm{C}$ & $35^{\circ} \mathrm{C}$ \\
\hline & 10 & 11 & 12 & 13 & 14 & 15 & 16 \\
\hline$d, \mathrm{~mm} / d, \mathrm{~mm}$ & 0.917 & 0.545 & 0.324 & 0.193 & \multicolumn{3}{|c|}{0.113} \\
\hline$d_{e}, \mathrm{MM} / d_{e}, \mathrm{~mm}$ & 0.637 & 0.471 & 0.303 & 0.172 & \multicolumn{3}{|c|}{0.099} \\
\hline$P$ & 0.389 & 0.383 & 0.380 & 0.378 & \multicolumn{3}{|c|}{0.368} \\
\hline$\eta \cdot 10^{-3}, \mathrm{~Pa} \cdot \mathrm{s}$ & \multicolumn{4}{|c|}{1} & 1.52 & 1.002 & 0.723 \\
\hline$\rho_{m}, \mathrm{~kg} / \mathrm{m}^{3}$ & 2011 & 2021 & 2026 & 2029 & 2050 & 2048 & 2046 \\
\hline$\kappa_{0} \cdot 10^{-11}, \mathrm{~m}^{2}$ & 26.7 & 14.6 & 6.06 & 1.94 & \multicolumn{3}{|c|}{0.6423} \\
\hline$T[8,9]$ & 1.58 & 1.58 & 1.58 & 1.58 & \multicolumn{3}{|c|}{1.58} \\
\hline$<\alpha_{\mathrm{s} 0}>\mathrm{dB} / \mathrm{m} / \mathrm{kHz}$ & $12.7 \pm 2.5$ & $13.7 \pm 2.7$ & $17.9 \pm 4.5$ & $20.4 \pm 4.0$ & $9.46 \pm 0.8$ & $9.85 \pm 0.33$ & $15.3 \pm 1.4$ \\
\hline$\alpha_{\mathrm{p} 0}, \mathrm{~dB} / \mathrm{m} / \mathrm{kHz}$ & no data & no data & no data & no data & no data & no data & no data \\
\hline$\gamma_{\mathrm{s}} \cdot 10^{6}, \mathrm{~Pa}$ & 27.5 & 25.1 & 23.0 & 20.1 & 62.8 & 47.1 & 31.6 \\
\hline$\gamma \cdot 10^{6}, \mathrm{~Pa}$ & no data & no data & no data & no data & no data & no data & no data \\
\hline$m$ & 0.0217 & 0.0197 & 0.020 & 0.0212 & 0.048 & 0.041 & 0.029 \\
\hline$n$ & no data & no data & no data & no data & no data & no data & no data \\
\hline$\varphi_{\mathrm{s}} ; \varphi_{\mathrm{p}}$ & 0.389 & 0.383 & 0.380 & 0.378 & 0.05 & 0.05 & 0.368 \\
\hline$d_{e} / a_{\mathrm{s}}$ & 20 & 23 & 23 & 14 & \multicolumn{3}{|c|}{15} \\
\hline$d_{e} / a_{\mathrm{p}}$ & no data & no data & no data & no data & \multicolumn{3}{|c|}{ no data } \\
\hline
\end{tabular}

$\mathrm{N}$ o t e. The column number and the color of a numeral denoting the number correspond to the number and color of the graph in Fig. 2 and 3. Legend: $d$ is the average grain size determined by the sieve analysis; $d_{e}$ is the equivalent grain size determined from the measured steady-state permeability of the medium; $P$ is the static porosity; $\eta$ is the pore fluid dynamic viscosity; $\rho_{m}$ is the equilibrium density of a saturated medium; $\kappa_{0}$ is the steady-state medium permeability; $T$ is the tortuosity coefficient; $\left\langle\alpha_{\mathrm{s} 0}\right\rangle$ is the measured frequency-average attenuation coefficient; $\alpha_{\mathrm{s} 0}$ is the measured attenuation coefficient; $\gamma_{\mathrm{s}}$ is the shear rigidity coefficient; $\gamma$ is the compressional rigidity coefficient; $m$ is the shear strain hardening exponent; $n$ is the compressional strain hardening exponent; $\varphi_{\mathrm{s}}, \varphi_{\mathrm{p}}$ are the shear and compressional effective porosities; $a_{\mathrm{s}}, a_{\mathrm{p}}$ are the pore sizes during propagation of the shear and compressional waves.

In dry media, the experiments reveal a constant low and weakly dependent on the size and shape of granules, specific attenuation coefficient $\alpha_{\mathrm{s} 0}(\mathrm{~dB} /(\mathrm{m} \cdot \mathrm{kHz})$, i.e. a linear frequency dependence $\alpha_{s}=\alpha_{s 0} \cdot f^{1}$. The shear wave velocity turns out to be higher than in a saturated medium and depends on the size and shape of the granules (black circles and diamonds, graphs 5, 7 in Fig. 2, a, 2, b). Such a relationship between the velocity and attenuation confirms the existence of an unambiguous conservative-dissipative relationship between them: the higher the velocity, the lower the attenuation. Saturation of the medium with fluid decreases the shear wave velocity and increases the attenuation (blue diamonds and light blue 
circles, graphs 6, 8 in Fig. 2, $a, 2, b$ ). The attenuation coefficient becomes frequency dependent.

In D. Bell's experiments, the longitudinal wave velocity and attenuation were also measured. So, it is possible to compare the compressional ( $n$ is the compressional strain hardening exponent, $\gamma$ is the compressional rigidity modulus $[12,13])$ and the shear characteristics of intergranular friction. If when the medium is saturated with fluid, the shear wave velocity decreases and the attenuation increases, then in the longitudinal wave, on the contrary, in a watersaturated medium, the speed of sound $c_{\mathrm{p}}$ is much higher $\left(c_{\mathrm{psat}} / c_{\mathrm{pdry}}\right.$ are $1740 / 220 \mathrm{~m} / \mathrm{s}$ in sand and 1810/150 m/s in beads), and the attenuation is much less than in dry one. Shear $\left(\gamma_{\mathrm{s}}\right)$ intergranular rigidity is much higher than compressional $(\gamma)\left(\gamma_{\text {sdry }}>\gamma_{\text {dry }}\right)$ one in a dry medium and much less in a saturated one $\left(\gamma_{\text {ssat }}<<\gamma_{\text {sat }}\right)$. The latter is explained by the addition of elasticity of the fluid trapped in the cracks between the granules. In dry media, the compressional $(n)$ strain hardening exponent is much higher than the shear (m) one: $n_{\text {dry }}>m_{\text {dry }}$; in saturated media $n_{\text {sat }}>m_{\text {sat, }}$ but these values are comparable. Recalling that the strain hardening exponent characterizes the nonlinearity of the medium, allowing even the inaccuracy of D. Bell's measurements (with respect to the longitudinal wave), it can be concluded that a dry medium turns out to be soft and substantially nonlinear under compression, but rigid and linear under shear. Saturation of the medium with fluid decreases shear micro friction, but increases the shear nonlinearity and multiplies the compression rigidity. The effect of saturation on compressional nonlinearity ( $n_{\text {dry }} \rightarrow n_{\text {sat }}$ transition) is much more complex. If the fluid viscosity is low (water), the compressional nonlinearity decreases; if the fluid is a viscous oil (see the description of the experiment with glass beads in oil [12, 13]), the nonlinearity becomes extremely high.

Now we are to pay attention to the influence of the shape of the granules. When the granules are in a dry state, the attenuation coefficient is almost independent of the granule shape (see graphs 5, 7 in Fig. 2, b). As seen from Fig. 2, a, 2, $c$ and tables, the shear intergranular rigidity determines the wave velocity. Comparing the results of B. Brunson's measurements in sieved and unsieved sand at the same speed (see graphs 2, 4 in Fig. 2, a), it can be seen that a greater of strain hardening (i.e. greater nonlinearity) is found in unsieved angular sands.

Fig. 2, $a, 2, b$ include the measurement results obtained in marine experiments. The SAX99 result confirms the quality of measurements by B. Brunson and D. Bell and the applicability of the GSEDs model to real marine sediments. Indeed, the experimental points corresponding to the SAX99 measurements (graph 9 in Fig. 2, $a, b$ ) are located between the graphs made according to the results of measurements by B. Brunson and D. Bell. This corresponds to the depth of the SAX99 sensors in the bottom $(30 \mathrm{~cm})$. Measurements at the lowest frequencies confirm both the "linear" and "non-linear" points of view, and most importantly, confirm the applicability of the GSEDs model in the region of the lowest frequencies.

Further the results of experiments by M. Kimura [8, 9] with sorted sand with various granule sizes (Fig. 2, c, 2, $d$ ) are considered. Matching parameters are focused on the $12 \mathrm{kHz}$ frequency and are selected so as to fit into the confidence intervals as much as possible. As can be seen from the table, the value of the strain hardening PHYSICAL OCEANOGRAPHY VOL. 28 ISS. 1 (2021) 
exponent is almost constant, the shear wave velocity is determined by the intergranular rigidity increasing proportionally to the size of the sand grains. The dependence of the attenuation coefficient on the granule size in the high-frequency region is determined by the change in the $\alpha_{s 0}(f)$ curve shape with an upward shift of the transient frequency, i.e. by the influence of the fluid. Note that for coarse sand (see graph 10 in Fig. 2, d) at high frequencies, the theoretical attenuation approaches its lower "dry" limit (graph 5 in Fig. 2, b). The latter contradicts the conclusions made in [24], where an increase in the shear wave attenuation with enlargement of the granules was noted (the measurement frequency was $100 \mathrm{kHz}$ ). A change in the attenuation $\leftrightarrow$ grain size ratio and negative dispersion at high frequencies are also seen in Fig. 2, $c, 2, d$, however, the wavelength here already becomes comparable to the particle diameter, therefore, an additional loss mechanism - the scattering is activated.

Comparison of the graphs in Fig. 2, $b, 2$, d, seems to convincing that in watersaturated sands the frequency dependence of the attenuation $\alpha_{s}(f)$ is nonlinear, and the $\mathrm{Q}$ factor of the medium and the exponent $\delta$ in the power law of attenuation are not constant, which is argued by R.D. Stoll, M. Kimura and N. Chotiros.

However, to throw doubt again, upon the validity of this conclusion is forced by $\mathrm{M}$. Kimura's experiments with the fine sand at different temperatures (Fig. 2, $e$, 2 , $f$; table). It is noteworthy that in this experiment the value of porosity is the lowest of all found in the works of M. Kimura. As is seen, at two low temperatures the attenuation coefficient does not depend on the frequency, the $\mathrm{Q}$ factor is constant (graphs 14, 15 in Fig. 2, e, 2, f). When heated, possibly due to thermal expansion of the sand or softening and changes in the shape of the polyethylene cylinders which the sand was packed in, the pore structure and/or the properties of contacts between the granules change, the attenuation coefficient increases and the quality factor deviates from a constant. Let us also pay attention to the fact that the shear wave velocity in the cold medium in this experiment is the highest, and in the hot one it approaches the velocities recorded at high porosities of the medium (Fig. 2, $c$ ).

Discussion and conclusion. Dry unconsolidated media such as sands confirm the property of constant $\mathrm{Q}$ factor. The specific attenuation coefficient during shear wave propagation weakly depends on the shape of the granules and approaches the lower limit $\alpha_{\mathrm{s} 0 \mathrm{dry}} \approx 7 \mathrm{~dB} /(\mathrm{m} / \mathrm{kHz})$. This limit possibly reflects the physical nature of shear intergranular friction and strain hardening. The shear wave propagation speed, on the contrary, strongly depends on the compaction of the medium (porosity and coordination number) and on the shape of the granules. Microasperities, adhering to each other during shear, could form a kind of transverse elastic skeletal frame, through which shear strain is transmitted. It determines the high shear wave velocity. The presence or absence of a skeletal frame does not affect the dissipative properties of grain-to-grain shearing. On the other hand, the formed skeletal frame blocks the cross-section of the pores and prevents the global movement of the fluid.

Saturation of the medium with fluid leads to dissipative effects such as a decrease in the shear wave velocity and an increase in attenuation. In well compacted media, where the skeleton has formed, the attenuation slightly increases 
above the lower limit of $\alpha_{\text {sodry }}$, which confirms the absence of viscous losses (graphs 14, 15 in Fig. 2, e, 2, f). In loose, weakly compacted media, viscous losses appear even at high $(20 \mathrm{kHz})$ frequencies and increase the attenuation coefficient in proportion to the medium noncompactness.

The mechanisms of interaction between granules and between granules and the movable fluid during compression and shear are fundamentally different.

A dry medium under compression shows a lower intergranular rigidity than under shear, which significantly depends on the roughness of the granules. Saturation of pores with a low-viscosity fluid (water) reduces the nonlinearity of the medium during compression (similar to an additional linear dashpot), saturation with a viscous fluid (oil) increases extremely. The nature of the saturation effect on the power of the medium compressional nonlinearity (strain hardening) depends on the roughness of the granules. If the intergranular contacts are acute-angled (Bell sand), the main contribution is made by contact nonlinearity; if they are round (Bell beads), the contact nonlinearity is replaced by the nonlinearity of the fluid film.

Saturation of pores with water reduces the shear intergranular rigidity (like a lubricant), but increases the shear nonlinearity of the medium as a whole.

In the present paper, the pores were represented in the form of cylindrical tubes, as in $[12,13]$. The same pore model for compression and shear resulted in the same correction function (5). If the pore shape is indeed the same, then the properties of the pore space during compression and shear are different. For a compressional wave, the estimate of the effective pore size (9) $a_{\mathrm{p}}=d_{e} / 7.35$ is consistently confirmed. For a shear wave, the estimate is different: $a_{\mathrm{s}}=d_{e} / 23$ for coarse sands and $a_{\mathrm{s}}=d_{e} / 15$ for fine sands (see the table for the $d_{e} / a_{\mathrm{s}}$ and $d_{e} / a_{\mathrm{p}}$ ratios). It is noteworthy that the effective shear porosity in almost all cases coincides with the static porosity $(P)$.

The main question posed in the article: "How does the shear wave attenuation coefficient $\left(\alpha_{s}=\alpha_{s 0} f^{f}\right)$ depend on frequency: linearly or nonlinearly?". And the best answer is found in Fig. 3, showing the frequency dependences of the exponent $\delta$, modeled for all the experiments considered above, if the power law of attenuation is written in the form $\alpha_{\mathrm{s}}=C(f) \cdot f^{\delta}, C(f) \neq \alpha_{\mathrm{s} 0}$. The exponent is calculated using the formula $\delta_{\mathrm{s}}=\frac{\partial \alpha_{\mathrm{s}} / \partial f}{\alpha_{\mathrm{s} 0}}$.

If for a compression wave the exponent $\delta_{\mathrm{p}}$ varies in the range from 0.7 to 1.7 [13], then for a shear wave it ranges within smaller limits, from 0.75 to 1.4 . Thus, it can be concluded that the effect of the relative fluid motion on the acoustic properties of the shear wave in sandy sediments should be considered as a borderline phenomenon. In compacted, consolidated sediments, viscous losses will not appear. In the sediments of the uppermost layer of the sea bottom, disturbed by surface waves, tidal currents and shipping, viscous losses will make a significant contribution, increasing the attenuation by $20 \mathrm{~dB} /(\mathrm{m} \cdot \mathrm{kHz})$ in the vicinity of a frequency of about $5 \mathrm{kHz}$ and by $10 \mathrm{~dB} /(\mathrm{m} \cdot \mathrm{kHz})$ at high frequencies for shear waves of $20 \mathrm{kHz}$. Accordingly, the shear wave velocity will also decrease. 

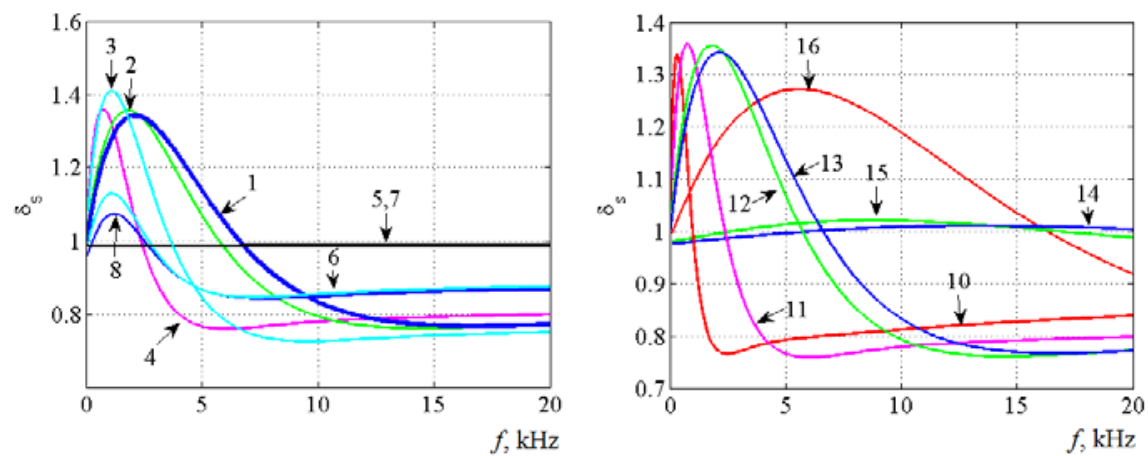

F i g. 3. Frequency dependence of the exponent $\delta_{\mathrm{s}}$ in the power law of attenuation: on the left - graphs 1-7; on the right - graphs 10-16 (the calculation graphs are indicated by the numbers just as in Fig. 2)

Eventually, during the propagation of a shear wave sandy sea sediments show a lower power of strain hardening (nonlinearity) than during the propagation of a compression wave, i.e., they exhibit the properties of a medium approaching elastic, in which the interaction between granules is described by Hooke's law.

\section{REFERENCES}

1. Jackson, D.R. and Richardson, M.D., 2007. High-Frequency Seafloor Acoustics. New York: Springer, 616 p. https://doi.org/10.1007/978-0-387-36945-7

2. Hovem, J.M., Richardson, M.R. and Stoll, R.D., Eds., 1991. Shear Waves in Marine Sediments. Dordrecht, Netherlands: Springer, 418 p. https://doi.org/10.1007/978-94-0113568-9

3. Kibblewhite, A.C., 1989. Attenuation of Sound in Marine Sediments: A Review with Emphasis on New Low-Frequency Data. The Journal of the Acoustical Society of America, 86(2), pp. 716-738. https://doi.org/10.1121/1.398195

4. Bowles, F.A., 1997. Observations on Attenuation and Shear-Wave Velocity in Fine-Grained, Marine Sediments. The Journal of the Acoustical Society of America, 101(6), pp. 3385-3397. https://doi.org/10.1121/1.419374

5. Hamilton, E.L., 1980. Geoacoustic Modeling of the Sea Floor. The Journal of the Acoustical Society of America, 68(5), pp. 1313-1340. https://doi.org/10.1121/1.385100

6. $\quad$ Stoll, R.D., 1989. Sediment Acoustics. New York: Springer, 153 p.

7. $\quad$ Brunson, B.A., 1983. Shear Wave Attenuation in Unconsolidated Laboratory Sediments: Ph. D. thesis. Corvalis, OR: Oregon State University, 253 p. Available at: https://ir.library.oregonstate.edu/downloads/9306t181w [Assessed: 19.06.2020].

8. Kimura, M., 2013. Shear Wave Speed Dispersion and Attenuation in Granular Marine Sediments. The Journal of the Acoustical Society of America, 134(1), pp. 144-155. https://doi.org/10.1121/1.4809679

9. Kimura, M., 2014. Grain-Size Dependence of Shear Wave Speed Dispersion and Attenuation in Granular Marine Sediments. The Journal of the Acoustical Society of America, 136(1), pp. EL53-EL59. https://doi.org/10.1121/1.4885478

10. Chotiros, N.P. and Isakson, M.J., 2014. Shear Wave Attenuation and Micro-Fluidics in Water-Saturated Sand and Glass Beads. The Journal of the Acoustical Society of America, 135(6), pp. 3264-3279. https://doi.org/10.1121/1.4874955

11. Chotiros, N.P., 2017. Acoustics of the Seabed as a Poroelastic Medium. Cham: Springer, 99 p. doi:10.1007/978-3-319-14277-7

12. Lisyutin, V.A., 2019. Generalized Rheological Model of the Unconsolidated Marine Sediments with Internal Friction and Effective Compressibility. Physical Oceanography, 26(1), pp. 77-91. doi:10.22449/1573-160X-2019-1-77-91 
13. Lisyutin, V.A. and Lastovenko, O.R., 2020. Assessing the Influence of Internal and Viscous Friction on Dispersion and Sound Attenuation in Unconsolidated Marine Sediments. Acoustical Physics, 66(4), pp. 401-415. https://doi.org/10.1134/S1063771020040065

14. Lisyutin, V.A., 2018. A Simple Acoustic Model of Unconsolidated Marine Sediments with Internal Friction and Viscous Dissipation. Ecological Bulletin of Research Centers of the Black Sea Economic Cooperation, 15(3), pp. 39-51. https://doi.org/10.31429/vestnik-153-39-51 (in Russian).

15. Buckingham, M.J., 2014. Analysis of Shear-Wave Attenuation in Unconsolidated Sands and Glass Beads. The Journal of the Acoustical Society of America, 136(5), pp. 2478-2488. https://doi.org/10.1121/1.4896468

16. Buckingham, M.J., 2000. Wave Propagation, Stress Relaxation, and Grain-To-Grain Shearing in Saturated, Unconsolidated Marine Sediments. The Journal of the Acoustical Society of America, 108(6), pp. 2796-2815. https://doi.org/10.1121/1.1322018

17. Pandey, V. and Holm, S., 2016. Connecting the Grain-Shearing Mechanism of Wave Propagation in Marine Sediments to Fractional Order Wave Equations. The Journal of the Acoustical Society of America, 140(6), pp. 4225-4236. https://doi.org/10.1121/1.4971289

18. Bedford, A., Costley, R.D. and Stern, M., 1984. On the Drag and Virtual Mass Coefficients in Biot's Equations. The Journal of the Acoustical Society of America, 76(6), pp. 1804-1809. https://doi.org/10.1121/1.391577

19. Buckingham, M.J., 2020. Wave Speed and Attenuation Profiles in a Stratified Marine Sediment: Geo-Acoustic Modeling of Seabed Layering Using the Viscous Grain Shearing Theory. The Journal of the Acoustical Society of America, 148(2), pp. 962-974. https://doi.org/10.1121/10.0001778

20. Brunson, B.A. and Johnson, R.K., 1980. Laboratory Measurements of Shear Wave Attenuation in Saturated Sand. The Journal of the Acoustical Society of America, 68(5), pp. 1371-1375. https://doi.org/10.1121/1.385104

21. Bell, D.W., 1979. Shear Wave Propagation in Unconsolidated Fluid Saturated Porous Media. Austin: The University of Texas at Austin.

22. Buckingham, M.J., 2007. On Pore-Fluid Viscosity and the Wave Properties of Saturated Granular Materials Including Marine Sediments. The Journal of the Acoustical Society of America, 122(3), pp. 1486-1501. https://doi.org/10.1121/1.2759167

23. Williams, K.L., Jackson, D.R., Thorsos, E.I., Tang, D. and Schock, S.G., 2002. Comparison of Sound Speed and Attenuation Measured in a Sandy Sediment to Predictions Based on the Biot Theory of Porous Media. IEEE Journal of Oceanic Engineering, 27(3), pp. 413-428. doi:10.1109/JOE.2002.1040928

24. Prasad, M. and Meissner, R., 1992. Attenuation Mechanisms in Sands; Laboratory Versus Theoretical (Biot) Data. Geophysics, 57(5), pp. 710-719. https://doi.org/10.1190/1.1443284

About the authors:

Victor A. Lisyutin - Associate Professor, Sevastopol State University (33, Universitetskaya St., Sevastopol, Russian Federation), Ph.D. (Phys.-Math.), vlisiutin@mail.ru

Olga R. Lastovenko - Associate Professor, Sevastopol State University (33, Universitetskaya, St., Sevastopol, Russian Federation), Ph.D. (Phys.-Math.)

Contribution of co-authors:

Victor A. Lisyutin - general concept, selection of experimental data, calculations, analysis and interpretation of results, writing the article text

Olga R. Lastovenko - assistance in the sexperimental data selection and in the computer software development, participation the article test preparation

All the authors have read and approved the final manuscript.

The authors declare that they have no conflict of interest. 